\title{
An 8-GS/s 200-MHz Bandwidth 68-mW $\Delta \Sigma$ DAC in 65-nm CMOS
}

Ameya Bhide, Omid Esmailzadeh Najari, Behzad Mesgarzadeh and Atila Alvandpour

\section{Linköping University Post Print}

\section{Tweet}

N.B.: When citing this work, cite the original article.

(C)2013 IEEE. Personal use of this material is permitted. However, permission to reprint/republish this material for advertising or promotional purposes or for creating new collective works for resale or redistribution to servers or lists, or to reuse any copyrighted component of this work in other works must be obtained from the IEEE.

Ameya Bhide, Omid Esmailzadeh Najari, Behzad Mesgarzadeh and Atila Alvandpour, An 8GS/s 200-MHz Bandwidth 68-mW $\Delta \Sigma$ DAC in 65-nm CMOS, 2013, IEEE Transactions on Circuits and Systems - II - Express Briefs, (60), 7, 387-391.

http://dx.doi.org/10.1109/TCSII.2013.2258272

Postprint available at: Linköping University Electronic Press http://urn.kb.se/resolve?urn=urn:nbn:se:liu:diva-96713 


\title{
An 8-GS/s 200-MHz Bandwidth 68-mW $\Delta \Sigma$ DAC in 65-nm CMOS
}

\author{
Ameya Bhide, Student Member, IEEE, Omid Esmailzadeh Najari, Student Member, IEEE, \\ Behzad Mesgarzadeh, Member, IEEE, and Atila Alvandpour, Senior Member, IEEE
}

\begin{abstract}
This paper presents an 8-GS/s, 12-bit input $\Delta \Sigma$ DAC with 200-MHz bandwidth in 65-nm CMOS. The high sampling rate is achieved by a two-channel interleaved MASH 1-1 digital $\Delta \Sigma$ modulator with 3-bit output, resulting in a highly digital DAC with only seven current cells. The two-channel interleaving allows the use of a single clock for both the logic and the final multiplexing. This requires each channel to operate at half the sampling rate, which is enabled by a high-speed pipelined MASH structure with robust static logic. Measurement results show that the DAC achieves 200-MHz bandwidth, 26-dB SNDR and -57-dBc IMD3, with a power consumption of $68-\mathrm{mW}$ at $1-\mathrm{V}$ digital and 1.2-V analog supplies. This architecture shows potential for use in transmitter baseband for wideband wireless communication.
\end{abstract}

Index Terms-Digital $\Delta \Sigma$ modulator (DDSM), digital-toanalog converter (DAC), time-interleaving, oversampling, MASH.

\section{INTRODUCTION}

$\mathbf{O}$ VERSAMPLED $\Delta \Sigma$ DACs have been reported in alldigital [1], "almost" digital [2] and RFDAC [3], [4] transmitters for UMTS, WLAN and WiMAX standards. Their use has been driven by the goal of a software defined radio wherein the bulk of the processing is moved into the digital domain in order to reduce the analog complexity and enable easy reconfiguration. The digital-to-analog conversion is pushed closer to the antenna and hence operates at a higher frequency compared to traditional transmitters. $\Delta \Sigma$ DACs thus become attractive in digital-centric transmitters because they greatly reduce the number of analog cells required compared to Nyquist DACs by using digital processing. Secondly, the oversampling relaxes the order of the reconstruction filter after the DAC. The example in Fig. 1 shows the difference between a traditional and a digital baseband transmitter that uses a $\Delta \Sigma$ DAC. It can be seen that the $\Delta \Sigma$ DAC, with much lesser number of current cells than a Nyquist DAC, relaxes the analog matching requirements. It extends the digital-analog boundary closer to the up-conversion mixer and operates at a frequency that depends on the radio standard, signal bandwidth and the required oversampling ratio (OSR).

High-speed high-data-rate communication requires increasingly larger bandwidths, such as the recent wideband standards for UWB [5] and 60-GHz [6] radios that have large RF

Manuscript received December 31, 2012. This work was supported by the Swedish Foundation for Strategic Research (SSF).

The authors are with Department of Electrical Engineering, Linköping University, SE-58183, Linköping, Sweden (email: ameya@ @isy.liu.se).

Copyright (c) 2013 IEEE. Personal use of this material is permitted. However, permission to use this material for any other purposes must be obtained from the IEEE by sending an email to pubs-permissions@ieee.org.

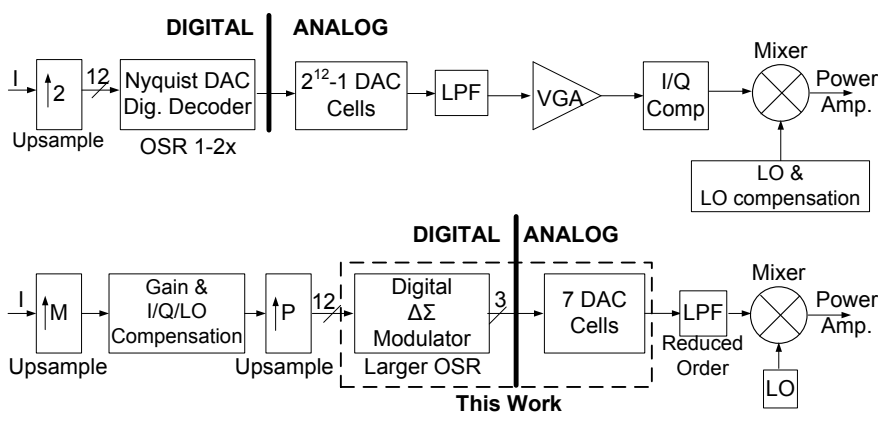

Fig. 1. Example of a Nyquist DAC in a traditional transmitter (top) and a $\Delta \Sigma$ DAC in a digital baseband transmitter (bottom). Only the I path is shown.

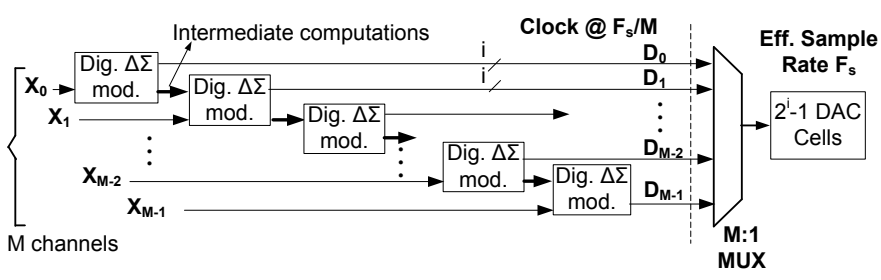

Fig. 2. Conceptual diagram of a M-channel interleaved DDSM $\Delta \Sigma$ DAC with sampling rate $F_{s}$. Each modulator operates at $F_{s} / M$ and the MUX at $F_{s}$.

bandwidths of $528 \mathrm{MHz}$ and $1.7 \mathrm{GHz}$ per channel respectively. However, the bandwidth of $\Delta \Sigma$ DACs reported so far has been limited to $100 \mathrm{MHz}$ (i.e. 200-MHz RF bandwidth when used in I \& Q paths of transmitters) [3]. Aiming to improve the bandwidth of $\Delta \Sigma$ DACs for wideband communication, this paper presents a $\Delta \Sigma$ DAC with $200-\mathrm{MHz}$ bandwidth in 65 nm CMOS.

OFDM modulation is commonly used for transmission at high data rates. If an OFDM signal is used with a modulation scheme like QPSK, 16-QAM or 64-QAM, then a SNR in the range of $25-35 \mathrm{~dB}$ is required from the DAC [3], [7]. Arriving at such an SNR for bandwidths over $200 \mathrm{MHz}$ requires DAC sampling rates exceeding $8 \mathrm{GS} / \mathrm{s}$. The speed of the Digital $\Delta \Sigma$ Modulator (DDSM) in the DAC then becomes the limiting factor for achieving this sampling rate when a conventional implementation is used.

Time-interleaved DDSM-based DACs are, therefore, required in order to relax the input data rate and the speed of the logic in the modulator. Fig. 2 shows this concept for a M-channel interleaved DDSM DAC. The digital processing contains $\mathrm{M}$ modulator sections operating at a relaxed $1 / \mathrm{M}^{\text {th }}$ of the desired sampling rate. The multiplexing of the channels just before the DAC is still at the full sampling rate [8].

Eight-channel interleaved DDSMs that operate at $2.5 \mathrm{GHz}$ 


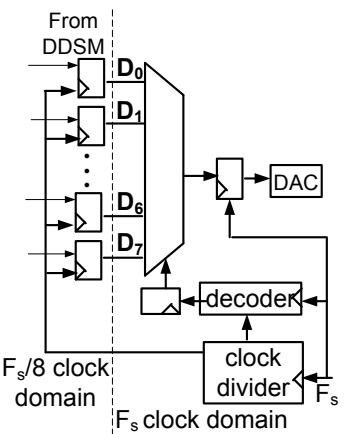

(a)

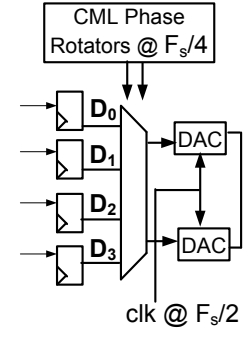

(b)

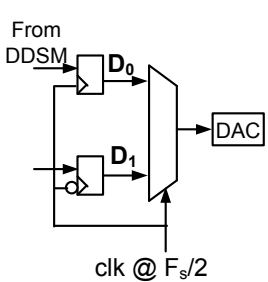

(c)
Fig. 3. (a) Eight-channel traditional multiplexing [9]. (b) Four-channel multiplexing with phase rotators and dual current cells [12]. (c) Two-channel multiplexing in this work.

have been previously described in [9] and [10]. However, the digital modulator in [9] does not address the DAC design, and in [10], the time-interleaving of the $\Delta \Sigma$ DAC is demonstrated by simulations. Furthermore, in [9] and [10], interleaving has been used with the aim of relaxing the logic speed, while its impact on the final full-speed multiplexing has not been considered due to the moderate speed of $2.5 \mathrm{GHz}$. However, for the $>8 \mathrm{GHz}$ speed targeted in this work, a high degree of interleaving complicates the routing and more importantly, it increases the timing complexity of the multiplexing which then requires accurate multi-phase clock generation. Therefore, a two-channel interleaving strategy is utilized in this work which enables a simplified multiplexer by allowing the use of a single clock for the logic as well as the multiplexing, while pushing the speed of the logic in each channel to $4 \mathrm{GHz}$.

This paper is organized as follows. Section II motivates the architecture choice. Section III describes the circuit design of the proposed $\Delta \Sigma$ DAC. The test chip and measurement results are presented in Section IV followed by the conclusion in Section V.

\section{II. $\Delta \Sigma$ DAC ARCHITECTURE}

A MASH architecture based $\Delta \Sigma$ DAC is suited for highspeed implementation because it is inherently stable, has a short integrator critical path and easily allows pipelining as compared to other typical modulator architectures [9]. Matlab simulations show that a MASH 1-1 DDSM DAC with seven thermometer-coded current cells (3-bit output) can provide a SNDR of $45-\mathrm{dB}$ at $200 \mathrm{MHz}$ bandwidth and 8-GS/s (OSR $=20$ ) with a current mismatch $(\sigma)$ of $3.6 \%$. A second order modulator presents only a moderate filtering complexity for suppressing the generated out-of-band quantization noise [2], [3]. For the chosen 1-1 MASH modulator, simulations show that a second order low pass filter can satisfy the spectral mask for the UWB standard in [5]. With these relaxed analog constraints, this becomes mainly a digital problem of designing a DDSM for such a high speed. For a conventional MASH 1-1 DDSM that uses static CMOS logic, the $8 \mathrm{GHz}$ speed is found to be outside the capability of a standard $65 \mathrm{~nm}$ technology node at $1 \mathrm{~V}$ even when extensive pipelining with 1bit integrators is utilized [4]. Hence, a time-interleaved MASH

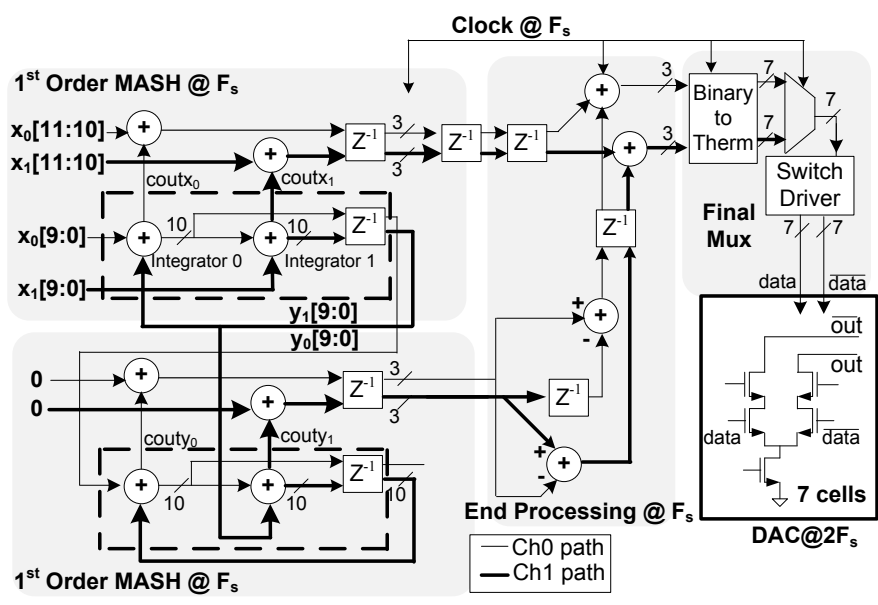

Fig. 4. Proposed two-channel interleaved second order MASH $\Delta \Sigma$ DAC with a $2 \mathrm{~F}_{\mathrm{s}}$ sampling rate. Critical path is enclosed by a dashed rectangle.

DDSM is necessary to relax the critical path and the clock rate.

In the $2.5 \mathrm{GHz}$ DDSM described in [9], eight-channel interleaving was chosen with the aim of relaxing the timing of the logic while minimizing the area. The multiplexer (MUX) was not a bottleneck at this speed and was easily achieved using a standard parallel-to-serial conversion. Fig. 3(a) shows this commonly used scheme where the eight individual channels working at an $F_{\mathrm{s}} / 8$ speed are retimed to $\mathrm{F}_{\mathrm{s}}$. It can be noted that the clock divider and channel select logic still operate at the full sampling rate, $F_{s}$. At $8 \mathrm{GHz}$, this multiplexing scheme becomes a bottleneck as meeting the clock divider and the channel select logic paths becomes a challenge with full-swing CMOS logic. In order to use this scheme at $8 \mathrm{GHz}$, a reduced-swing logic is required instead, e.g. CML. However, the reduced swing in the DAC switch drivers would then affect the DAC linearity [11].

Another multiplexing style (Fig. 3(b)), described in [12] for four channels, uses CML and analog phase rotators for setting the clock phases. In this MUX, the final latches are moved into the current cell itself and two current cells per bit are used. This scheme requires a modified current cell and a complicated routing with three times the number of wires going into the cell. The number of current cells also doubles, resulting in an overall increase of analog complexity.

The two MUX choices show that the increased relaxation in the logic timing resulting from an increased degree of interleaving comes at a cost of complicated MUX and current cell circuits. Hence, two-channel interleaving (Fig. 3(c)) is chosen in this work by giving a higher priority to the simplification of the MUX and the current cells. This two-channel approach has the following benefits. Firstly, the logic and the MUX now work with a single half-rate clock and secondly, it allows multiplexing with rail-to-rail swing and use of traditional current cells. However, this requires the logic in each channel to operate at $4 \mathrm{GHz}$, which is still a challenging task. This speed has been achieved by a careful integrator design that is tailored for the interleaved structure and is described in the following section. 


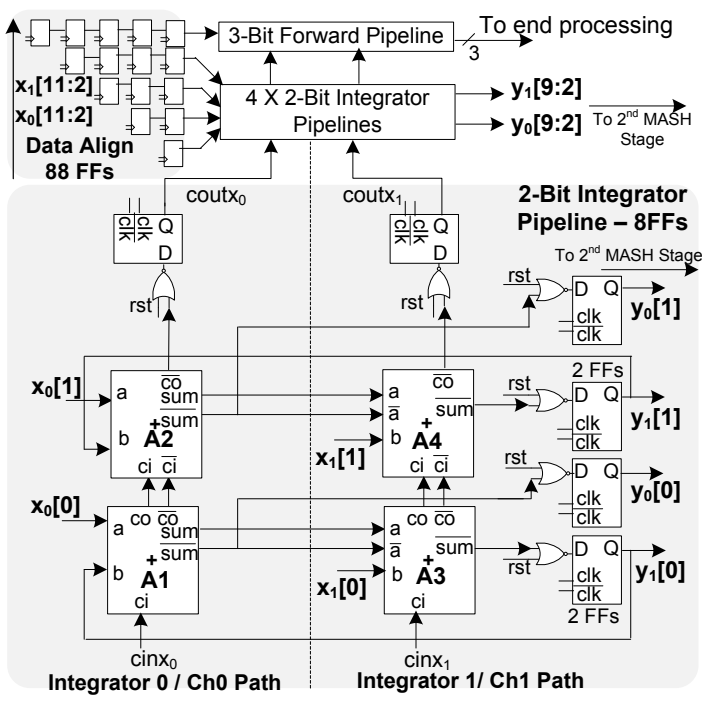

Fig. 5. Design of first order interleaved MASH DDSM. Each 2-bit integrator slice uses four 1-bit carry select adders and transmission gate FFs. Two critical paths exist here.

\section{III. $\Delta \Sigma$ DAC CIRCUIT DESIGN}

\section{A. DDSM design}

Fig. 4 shows the block diagram of the proposed 12-bit $\Delta \Sigma$ DAC that uses the two-channel interleaved MASH 11 architecture and seven current cells. Inputs $\mathrm{x}_{0}[11: 0]$ and $\mathrm{x}_{1}[11: 0]$, form the two half-rate channels and are the divided even and odd streams of the original data-rate. The 10-bit back-to-back integrators/adders, i.e. the feedback path in each of the first order MASH DDSMs form the critical path in the design (shown with a dashed rectangle). The carry generated from the integrators is added to the remaining two MSB input bits, $\mathrm{x}_{0}$ [11:10] and $\mathrm{x}_{1}$ [11:10]. Being in a forward path, this addition and also the end processing are not critical paths. Therefore, the main design problem becomes that of two 10-bit back-to-back adders that must operate at over $4 \mathrm{GHz}(250 \mathrm{ps}$ cycle time).

The aim of this work has also been to enable the high sampling rate by utilizing only robust static logic so as to avoid the lower noise margins that are associated with faster logic styles such as pre-charged domino, ratioed or pass transistor logic. Pipelining is essential for meeting the speed requirement. Furthermore, in order to maximize the speed of the pipeline and the number of bits that can be accommodated in each pipeline, an important observation about the two adders can be made. In the first adder (Integrator 0), both the carry chain and the sum delays are in the critical path but in the second adder (Integrator 1), only the carry chain is in the critical path. Hence, using the same adder cells for both the adders does not result in the fastest speed. Using this observation, all the non-critical nodes of both the adders are slowed down by reducing their drive strength. This helps to reduce the capacitance on the critical path and speed it up.

Fig. 5 shows the implementation of a first order twochannel interleaved MASH stage that is instantiated twice in the design. Only static CMOS logic and Transmission Gate Flip-flops (TGFF) are used. Each MASH consists of
TABLE I

PATH 1 AND 2 DELAY BREAKDOWNS. THE DELAYS ARE POST-LAYOUT SIMULATED AT TYPICAL CORNER, $1 \mathrm{~V}, 75^{\circ} \mathrm{C}$ AND $4.5 \mathrm{GHZ}$.

\begin{tabular}{|c|c|c|c|c|c|}
\hline \multicolumn{2}{|c|}{ Path 1 } & \multicolumn{3}{c|}{ Path 2 } \\
\hline Circuit & $\begin{array}{c}\text { Delay } \\
\text { Type }\end{array}$ & $\begin{array}{c}\text { Delay } \\
(\mathbf{p s})\end{array}$ & Circuit & $\begin{array}{c}\text { Delay } \\
\text { Type }\end{array}$ & $\begin{array}{c}\text { Delay } \\
(\mathbf{p s})\end{array}$ \\
\hline $\mathrm{y}_{1}[0] \mathrm{FF}$ & $\mathrm{clk} \rightarrow \mathrm{q}$ & 40 & $\mathrm{y}_{1}[0] \mathrm{FF}$ & $\mathrm{clk} \rightarrow \mathrm{q}$ & 40 \\
\hline Adder A1 & $\mathrm{b} \rightarrow \mathrm{co}$ & 60 & Adder $\mathrm{A} 1$ & $\mathrm{~b} \rightarrow \mathrm{sum}$ & 63 \\
\hline Adder A 2 & $\mathrm{ci} \rightarrow \mathrm{sum}$ & 27 & Adder $\mathrm{A} 3$ & $\mathrm{a} \rightarrow \mathrm{co}$ & 44 \\
\hline Adder A4 & $\mathrm{a} \rightarrow \overline{\mathrm{sum}}$ & 41 & Adder $\mathrm{A} 4$ & $\mathrm{ci} \rightarrow \overline{\mathrm{sum}}$ & 19 \\
\hline Reset NOR & in $\rightarrow$ out & 27 & Reset NOR & $\mathrm{in} \rightarrow$ out & 27 \\
\hline $\mathrm{y}_{1}[1] \mathrm{FF}$ & $\mathrm{T}_{\text {setup }}$ & 24 & $\mathrm{y}_{1}[1] \mathrm{FF}$ & $\mathrm{T}_{\text {setup }}$ & 24 \\
\hline Total Path 1 Delay (ps) & $\mathbf{2 1 9}$ & Total Path 2 Delay (ps) & $\mathbf{2 1 7}$ \\
\hline
\end{tabular}

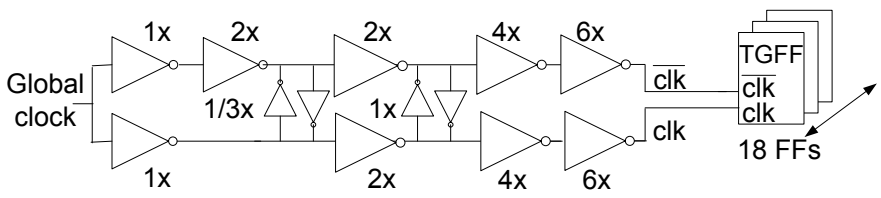

Fig. 6. Pseudo-differential clock driver used for local distribution. One clock driver is used per $18 \mathrm{FFs}$.

five pipeline stages of 2-bit integrators and one 3-bit forward pipeline stage. Each 2-bit integrator pipeline uses four 1-bit carry select adders. Adders A1-A2, together form Integrator 0 while A3-A4 form Integrator 1 . Based on the observations about the adders mentioned previously, all the four carry select adders are sized differently. There are two critical paths in each pipeline stage, from $\mathrm{y}_{1}[0] \mathrm{FF} \rightarrow \mathrm{A} 1 \rightarrow \mathrm{A} 2 \rightarrow \mathrm{A} 4 \rightarrow$ $\mathrm{NOR} \rightarrow \mathrm{y}_{1}$ [1] FF (Path 1) and $\mathrm{y}_{1}[0] \mathrm{FF} \rightarrow \mathrm{A} 1 \rightarrow \mathrm{A} 3 \rightarrow \mathrm{A} 4$ $\rightarrow$ NOR $\rightarrow \mathrm{y}_{1}[1]$ FF (Path 2). Table I shows the post-layout delay contributions from both the paths at the typical corner, $1 \mathrm{~V}$ supply and $75^{\circ} \mathrm{C}$. The table shows that this optimized 2bit structure supports a frequency greater than $4 \mathrm{GHz}$ and the delays in the two paths are nearly equalized.

Adder A1 is the slowest in the path as it generates both the sum and co (carry) complementary outputs with equal delay . It also internally generates the complementary inputs, which slows it by one additional inverter delay. In comparison, A2 has a faster $c i \rightarrow s u m$ delay as its $c i \rightarrow \overline{c o}$ delay is non-critical. On the same lines, A3 has a faster $a \rightarrow c o$ delay as its $a \rightarrow \overline{\text { sum }}$ delay is non-critical. A4 equalizes both the co and sum delays similar to A1, but is faster due to its lower fan-out. The NOR gate at the end of the adder path is an additional overhead required to synchronously reset the integrator at start-up.

\section{B. Clock Distribution}

In order to keep the TGFFs compact in size by avoiding a clock inverter inside the flip-flops, both the clock phases are provided from outside the FF. While the global clock distribution is single-ended, the pseudo-differential clock driver of Fig. 6 distributes the clocks with a 30 ps slope locally to the FFs. Each such clock driver is used for every $18 \mathrm{FFs}$ (fan-out of 3) in a $70 \mu \mathrm{m} \times 45 \mu \mathrm{m}$ area. This driver also minimizes the clk-clk overlap as both the clock phases have an equal load. 


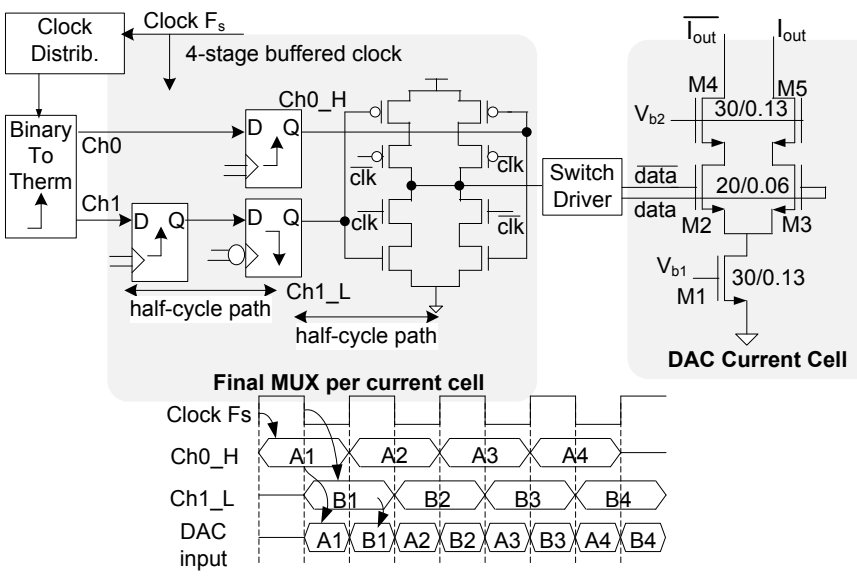

Fig. 7. Final MUX and DAC current cell with the timing diagram. Switch driver circuit is the same as the local clock driver of Fig. 6.

\section{Final Multiplexer and Current Cell Design}

Fig. 7 shows the static 2:1 MUX per cell. The data from the second channel is shifted by half a cycle prior to the MUX. The MUX is single-ended and the complementary DAC switch signals are generated with a $15 \mathrm{ps}$ slope after the MUX by a center-crossing pseudo-differential switch driver. The switch driver uses the same circuit as the local clock driver of Fig. 6. A high-crossing switch driver [11] that is modified for this MUX structure is required in the DAC, but such a driver is a challenge at $8 \mathrm{GS} / \mathrm{s}$ due to the high capacitance and contention at its cross-coupled nodes. Hence, the center-crossing driver is chosen to meet the speed and fast slope. The DAC is sensitive to a mismatch in driver rise and fall delays and requires a careful driver design. However, due to its pseudo-differential nature, a 3 ps mismatch is the smallest that could be achieved in this design and this is found to degrade the SNDR by $4 \mathrm{~dB}$ in post-layout simulation. The clock to the MUX bypasses the main clock distribution and uses a minimized buffering of four stages. The MUX is sensitive to the clock duty cycle and simulations show an SNDR reduction of $4 \mathrm{~dB}$ for every $1 \%$ variation from the desired $50 \%$ duty cycle.

The DAC current cell, also shown in Fig. 7 is designed for $0.3 \mathrm{~V}_{\mathrm{pp} \text {-diff }}$ swing with a $100 \Omega$ passive load and $1.2 \mathrm{~V}$ supply. The current source dimensions for the $3.6 \%$ mismatch requirement are derived using foundry matching parameters. The current cell has M4-M5 transistors as the cascode pair on top of the switching M2-M3 pair that is biased in the linear region and M1 as the current source. The seven current cells are laid out in a single column with dummies on either side to simplify the route matching between the switch driver and the switches.

\section{Measurement Results}

The proposed $\Delta \Sigma$ DAC is implemented in a standard $65 \mathrm{~nm}$ CMOS process and uses only general purpose low- $\mathrm{V}_{\mathrm{T}}$ (DDSM $\&$ DAC) and standard- $\mathrm{V}_{\mathrm{T}}$ devices (DAC). Fig. 8 shows the chip photograph. The active area of the DAC is $0.13 \mathrm{~mm}^{2}$. The test chip is directly wire-bonded to an FR4 PCB.

Fig. 9 shows the measurement setup used. A 12-bit input of 8192 sample length and frequency $F_{\text {in }}$ is sent in to the chip

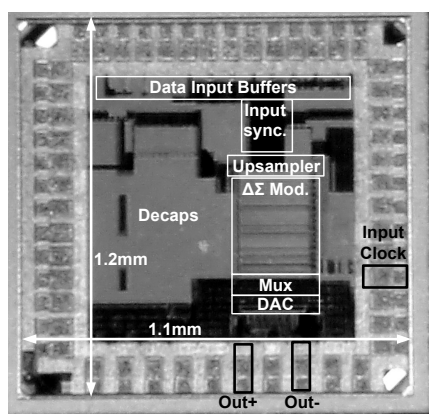

Fig. 8. Chip photograph of the implemented $\Delta \Sigma$ DAC

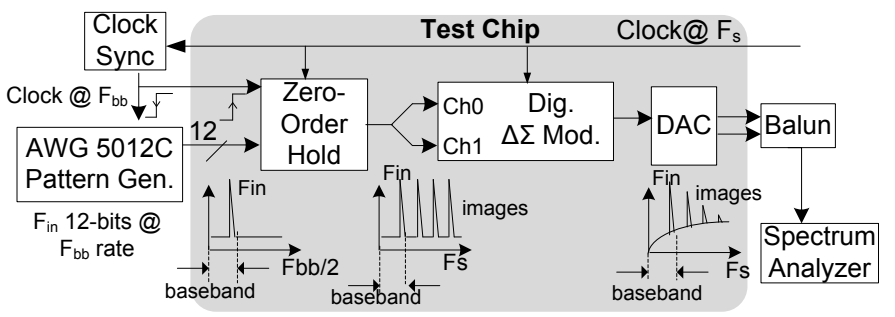

Fig. 9. Measurement setup for the $\Delta \Sigma$ DAC with the expected spectrum at output of every block. An upsampling filter is not used to simplify testing. Upsampling images in the output are out of the band of interest.

at a rate $\mathrm{F}_{\mathrm{bb}}$ using a Tektronix AWG5012C pattern generator. Internal to the chip, this data is upsampled to $F_{s}$ rate by a zero-order hold operation. In addition, the two DDSM channel inputs are also shorted together. The zero-hold operation and the shorted inputs, together results in upsampling of the input data by $2 \mathrm{~F}_{\mathrm{s}} / \mathrm{F}_{\mathrm{bb}}$ since the DAC effectively works at a $2 \mathrm{~F}_{\mathrm{s}}$ sampling rate. A FIR low-pass filter is not implemented to remove the upsampling images at the DDSM input. With this simplified setup, these unfiltered images appear at the DAC output also, but they lie outside the band of interest. In a real application, however, the upsampling images are filtered out prior to the DDSM. By keeping $F_{i n} \leq F_{b b} / 4$, the unfiltered images do not result in intermodulation products in the band of interest (DC to $\left.F_{i n}\right)$. The duty cycle of clock $F_{s}$ is tuned off-chip before the measurements.

Fig. 10 shows the measured single-ended output spectrum of a $200-\mathrm{MHz}$ full-scale single tone input at $8 \mathrm{GS} / \mathrm{s}$ operation of the $\Delta \Sigma$ DAC. With $F_{b b}=800 \mathrm{MHz}$ and $F_{s}=4 \mathrm{GHz}$, the main $200 \mathrm{MHz}$ tone and the 9 expected images are seen along with the noise shaping, thus demonstrating the desired DAC operation. The DAC achieves 26-dB SNDR for the $200 \mathrm{MHz}$ single tone input consuming $68 \mathrm{~mW}(40 \mathrm{~mW}-$ clocking, $23 \mathrm{~mW}$-logic/FF, $5 \mathrm{~mW}$-analog) from 1-V digital and $1.2-\mathrm{V}$ analog supplies. The measured IMD3 is $-57 \mathrm{dBc}$ (Fig. 11) for two $-6 \mathrm{dBFS}$ tones near $200 \mathrm{MHz}$ placed $2 \mathrm{MHz}$ apart.

Fig. 12 shows the simulated DAC output spectrum which has a SNDR of $42 \mathrm{~dB}$. The measured SNDR is found to be lower than the simulated value primarily due to a $10 \mathrm{~dB}$ loss resulting from a test setup limitation of synchronizing the $F_{s}$ and $F_{b b}$ clocks. The two clock sources have been locked to a common $50 \mathrm{MHz}$ reference, but they are still not truly synchronous. This results in an upsampling uncertainty in the 


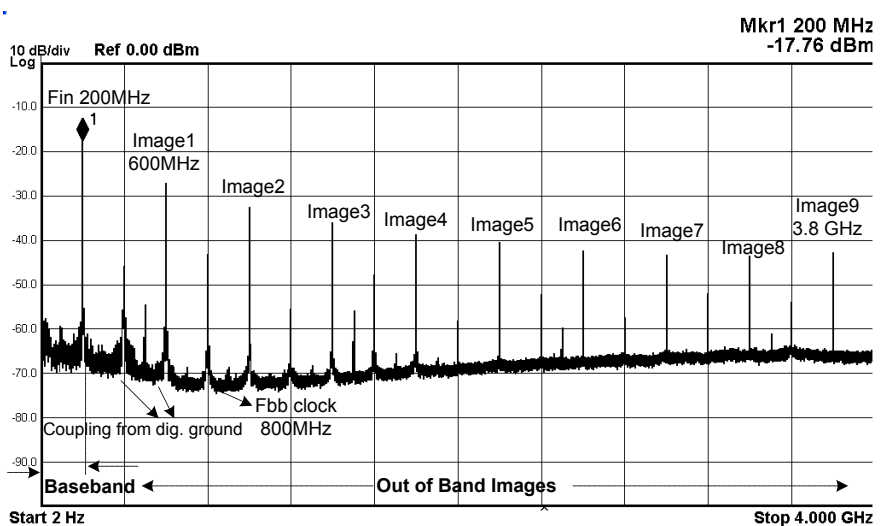

Start $2 \mathrm{~Hz}$ \#Res BW $300 \mathrm{kHz}$

\#VBW $1.1 \mathrm{kHz}$

Stop $4.000 \mathrm{GHz}$
Sweep $9.45 \mathrm{~s}(1001 \mathrm{pts})$

Fig. 10. Measured single-ended spectrum showing $8 \mathrm{GS} / \mathrm{s}$ operation with $\mathrm{F}_{\mathrm{s}}=4 \mathrm{GHz}, \mathrm{F}_{\mathrm{bb}}=800 \mathrm{MHz}$ and input frequency, $\mathrm{F}_{\mathrm{in}}=200 \mathrm{MHz}$. The noise shaping and the 9 out of band images can be seen.

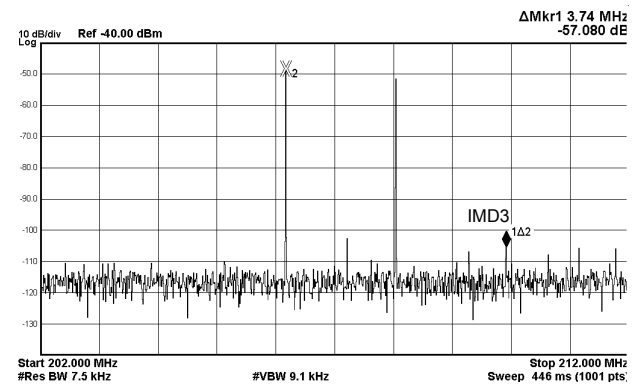

Fig. 11. Measured -57-dBc IMD3 with two -6 dBFS tones near $200 \mathrm{MHz}$ spaced $2 \mathrm{MHz}$ apart.

zero-hold operation and restricts the SNDR. The higher noise floor up to $800 \mathrm{MHz}$ in the measured spectrum results from this synchronization problem. Table II shows the comparison of this work with previous $\Delta \Sigma$ DACs having $>2.5 \mathrm{GHz}$ sampling rate.

\section{CONClusion}

This paper has presented a $8-\mathrm{GS} / \mathrm{s}$ 200-MHz bandwidth $\Delta \Sigma$ DAC with 26-dB SNDR, -57-dB IMD3 and 68-mW power consumption in $65 \mathrm{~nm}$ CMOS. The high sampling rate was achieved by a two-channel interleaved 4-GHz MASH 11 modulator structure. This allowed a single clock solution, thus simplifying the timing complexity of the final full-rate multiplexing. Using only seven current cells with relaxed matching requirements, this work demonstrates the potential of this predominantly digital DAC for use in baseband of transmitters for wideband wireless communication, e.g. UWB and $60-\mathrm{GHz}$ radios.

\section{ACKNOWLEDGMENT}

The authors thank B. Ygfors, Caltech AB for pattern generator support.

\section{REFERENCES}

[1] A. Frappe, A. Flament, B. Stefanelli, A. Kaiser and A. Cathelin, "An All-Digital RF Signal Generator Using High-Speed $\Delta \Sigma$ Modulators", IEEE J. Solid State Circuits, vol. 44, pp. 2722-2732, Oct. 2009.

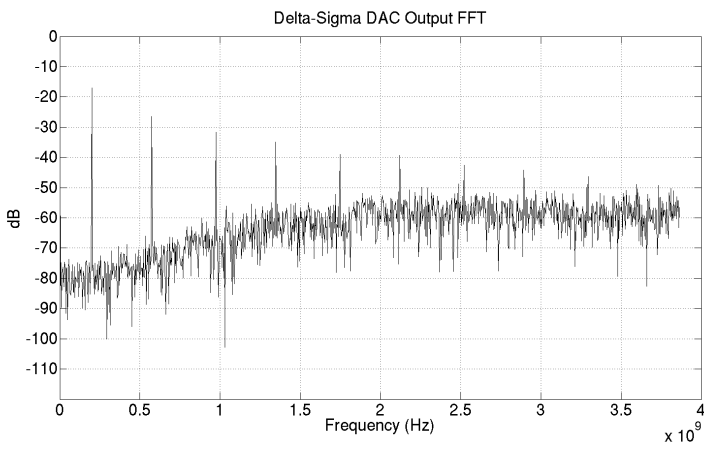

Fig. 12. Output spectrum obtained from simulation.

TABLE II

COMPARISON WITH $\Delta \Sigma$ DACS HAVING $>2.5$-GS/S SAMPLING RATE.

\begin{tabular}{|l|c|c|c|c|c|}
\hline Paper & {$[2]$} & {$[4]$} & {$[1]$} & {$[3]$} & $\begin{array}{c}\text { This } \\
\text { Work }\end{array}$ \\
\hline DAC Type & $\Delta \Sigma$ DAC & $\begin{array}{c}\Delta \Sigma \\
\text { RFDAC }\end{array}$ & $\Delta \Sigma$ DAC & $\begin{array}{c}\Delta \Sigma \\
\text { RFDAC }\end{array}$ & $\begin{array}{c}\Delta \Sigma \\
\text { DAC }\end{array}$ \\
\hline $\begin{array}{l}\text { Modulator } \\
\text { Type }\end{array}$ & $\begin{array}{c}\text { Error } \\
\text { Feedback }\end{array}$ & MASH & $\begin{array}{c}\text { Error } \\
\text { Feedback }\end{array}$ & MASH & $\begin{array}{c}\text { Interleaved } \\
\text { MASH }\end{array}$ \\
\hline Technology & $90 \mathrm{~nm}$ & $65 \mathrm{~nm}$ & $90 \mathrm{~nm}$ & $0.13 \mu \mathrm{m}$ & $65 \mathrm{~nm}$ \\
\hline Input Bits & 10 & 5 & 13 & 12 & 12 \\
\hline Output Bits & 3 & 3 & 1 & 3 & 3 \\
\hline Order & 2 & 3 & 3 & 2 & 2 \\
\hline $\begin{array}{l}\text { Sampling Rate } \\
\text { (GS/s) }\end{array}$ & 3.6 & 5.4 & 4 & 2.6 & 8 \\
\hline $\begin{array}{l}\text { Bandwidth } \\
\text { (MHz) }\end{array}$ & 10 & 10 & 50 & 100 & 200 \\
\hline SNDR (dB) & 70 & - & 53 & 30 & 26 \\
\hline IMD3 (dBc) & -70 & - & - & -51 & -57 \\
\hline Area (mm ${ }^{2}$ ) & $0.06^{*}$ & - & $<0.15$ & $<0.11^{*}$ & 0.13 \\
\hline Power (mW) & 16 & $>50^{*}$ & $54^{*}$ & $40^{*}$ & 68 \\
\hline $\begin{array}{l}\text { Swing } \\
\text { (V) }\end{array}$ & $0.3^{*}$ & - & 1.3 & 0.35 & 0.3 \\
\hline
\end{tabular}

Estimated. ${ }^{1}$ [4] is a hybrid $\Delta \Sigma$ and Nyquist RFDAC.

[2] P. Seddighrad, A. Ravi, M. Sajadieh, H. Lakdawala and K. Soumyanath, "A $3.6 \mathrm{GHz} 16 \mathrm{~mW} \triangle \Sigma$ DAC for a $802.11 \mathrm{n} / 802.16 \mathrm{e}$ transmitter with $30 \mathrm{~dB}$ digital power control in 90nm CMOS“, Proc. IEEE European Solid-State Circuits Conf., 2008, pp. 202-205.

[3] A. Jerng and C. Sodini, "A Wideband $\Delta \Sigma$ Digital-RF Modulator for High Data Rate Transmitters", IEEE J. Solid State Circuits, vol. 42, pp. 1710-1722, Aug. 2007.

[4] A. Pozsgay, T. Zounes, R. Hossain, M. Boulemnakher, V. Knopnik and S. Grange, "A Fully Digital 65nm CMOS Transmitter for the 2.4-to2.7GHz WiFi/WiMAX Bands using 5.4GHz $\triangle \Sigma$ RF DACs", IEEE. Int. Solid-State Circ. Conf. Dig. Tech. Papers, 2008, pp. 360-619.

[5] High Rate Ultra Wideband PHY and MAC Standard, ECMA-368, Dec. 2008 [Online]. Available: www.ecma-international.org.

[6] WirelessHD Specification Version 1.1 Overview, May. 2010 [Online]. Available: www.wirelesshd.org.

[7] Q. Gu, RF System Design of Transceivers for Wireless Communication, Springer, 2005.

[8] S. Balasubramanian et al., "Systematic Analysis of Interleaved Digitalto-Analog Converters", IEEE. Trans. Circuits Systems-II:Express Briefs, vol. 58, pp. 882-886, Dec. 2011.

[9] P. Madoglio et al., "A 2.5-GHz, 6.9-mW, 45-nm-LP CMOS, $\Delta \Sigma$ Modulator Based on Standard Cell Design With Time-Interleaving", IEEE J. Solid State Circuits, vol. 45, pp. 1410-1420, July 2010.

[10] J. Pham and A. C. Carusone, "A Time-Interleaved $\Delta \Sigma$-DAC Architecture Clocked at the Nyquist Rate", IEEE. Trans. Circuits SystemsII:Express Briefs, vol. 55, pp. 858-862, Sept. 2008.

[11] C. Lin et al., "A 12 bit 2.9 GS/s DAC With IM3 <-60 dBc Beyond $1 \mathrm{GHz}$ in $65 \mathrm{~nm}$ CMOS", IEEE J. Solid State Circuits, vol. 44, no. 12. pp. $3285-3293$, Dec. 2009.

[12] J. Savoj, A. Abbasfar, A. Amirkhany, M. Jeeradit, and B. Garlepp. "A 12-GS/s Phase-Calibrated CMOS Digital-to-Analog Converter for Backplane Communications", IEEE J. Solid State Circuits, vol. 43, no. 5. pp. $1207-1216$, May 2008. 